\title{
Nucleon structure from 2+1 flavor lattice QCD near the physical point
}

\author{
Natsuki Tsukamoto ${ }^{1, \star}$, Ken-Ichi Ishikawa ${ }^{2,3}$, Yoshinobu Kuramashi ${ }^{4,5,3}$, Shoichi Sasaki ${ }^{1,3}$, and \\ Takeshi Yamazaki ${ }^{5,4,3}$ for PACS Collaboration \\ ${ }^{1}$ Department of Physics, Tohoku University, Sendai, Miyagi 980-8578, Japan \\ ${ }^{2}$ Department of Physics, Hiroshima University, Higashi-Hiroshima, Hiroshima 739-8526, Japan \\ ${ }^{3}$ RIKEN Advanced Institute for Computational Science, Kobe, Hyogo 650-0047, Japan \\ ${ }^{4}$ Center for Computational Sciences, University of Tsukuba, Tsukuba, Ibaraki 305-8577, Japan \\ ${ }^{5}$ Faculty of Pure and Applied Sciences, University of Tsukuba, Tsukuba, Ibaraki 305-8571, Japan
}

\begin{abstract}
We present an update on our results of nucleon form factors measured on a large-volume lattice $(8.1 \mathrm{fm})^{4}$ at almost the physical point in $2+1$ flavor QCD. The configurations are generated with the stout-smeared $O(a)$ improved Wilson quark action and Iwasaki gauge action at $\beta=1.82$, which corresponds to the lattice spacing of 0.085 $\mathrm{fm}$. The pion mass at the simulation point is about $145 \mathrm{MeV}$. We determine the isovector electric radius and magnetic moment from nucleon electric $\left(G_{E}\right)$ and magnetic $\left(G_{M}\right)$ form factors. We also report on preliminary results of the axial-vector $\left(F_{A}\right)$, induced pseudo-scalar $\left(F_{P}\right)$ and pseudo-scalar $\left(G_{P}\right)$ form factors in order to verify the axial WardTakahashi identity in terms of the nucleon matrix elements, which may be called as the generalized Goldberger-Treiman relation.
\end{abstract}

\section{Introduction}

In general, four form factors appear in the nucleon matrix elements of the weak current. Here, for example, we consider the matrix element for neutron beta decay. In this case, the vector and axialvector currents are given by $V_{\alpha}^{+}(x)=\bar{u}(x) \gamma_{\alpha} d(x)$ and $A_{\alpha}^{+}(x)=\bar{u}(x) \gamma_{\alpha} \gamma_{5} d(x)$, and then the general form of the matrix element for neutron beta decay is expressed by both the vector and axial-vector transitions:

$$
\left\langle p\left|V_{\alpha}^{+}(x)+A_{\alpha}^{+}(x)\right| n\right\rangle=\bar{u}_{p}\left(O_{\alpha}^{V}(q)+O_{\alpha}^{A}(q)\right) u_{n} e^{i q \cdot x},
$$

where $q=P_{n}-P_{p}$ is the momentum transfer between the neutron $(n)$ and proton $(p)$. The vector $\left(F_{V}\right)$ and induced tensor $\left(F_{T}\right)$ form factors are introduced for the vector matrix element:

$$
O_{\alpha}^{V}(q)=\gamma_{\alpha} F_{V}\left(q^{2}\right)+\sigma_{\alpha \beta} q_{\beta} F_{T}\left(q^{2}\right)
$$

and also the axial-vector $\left(F_{A}\right)$ and induced pseudo-scalar $\left(F_{P}\right)$ form factors for the axial-vector matrix element:

$$
O_{\alpha}^{A}(q)=\gamma_{\alpha} F_{A}\left(q^{2}\right)+i q_{\alpha} \gamma_{5} F_{P}\left(q^{2}\right)
$$

`Speaker, e-mail: tsukamoto@nucl.phys.tohoku.ac.jp 
The vector part of weak processes is related to the nucleon's electromagnetic matrix element through an isospin rotation if heavy flavor contributions are ignored under the exact isospin symmetry $\left(m_{u}=m_{d}\right)$. The iso-vector electric $G_{E}\left(q^{2}\right)$ and magnetic $G_{M}\left(q^{2}\right)$ Sachs form factors are given by appropriate linear combinations of $F_{V}\left(q^{2}\right)$ and $F_{T}\left(q^{2}\right)$ as

$$
G_{E}\left(q^{2}\right)=F_{V}\left(q^{2}\right)-\frac{q^{2}}{2 M_{N}} F_{T}\left(q^{2}\right) \text { and } G_{M}\left(q^{2}\right)=F_{V}\left(q^{2}\right)+2 M_{N} F_{T}\left(q^{2}\right),
$$

where $M_{N}$ denotes the nucleon mass. Both form factors $G_{E / M}\left(q^{2}\right)$ can be expanded in powers of $q^{2}$ as

$$
G_{E(M)}\left(q^{2}\right)=G_{E(M)}(0)\left(1-\frac{q^{2}}{6}\left\langle r^{2}\right\rangle_{E(M)}+\frac{q^{4}}{120}\left\langle r^{4}\right\rangle_{E(M)}+O\left(q^{6}\right)\right),
$$

where the electric (magnetic) root-mean-square (RMS) radius $r_{E(M)}=\sqrt{\left\langle r^{2}\right\rangle_{E(M)}}$ can be evaluated from the slope of the form factors at zero momentum transfer. The value of $G_{E}(0)\left(G_{M}(0)\right)$ corresponds to a difference between charges (magnetic moments) of the proton and neutron states.

The latest lattice calculations of the nucleon structure have been carried out with increasing accuracy [1-5]. It seems that there is still a gap between experimentally known values and the lattice results, especially for the electric-magnetic nucleon radii and the magnetic moment. Our preliminary results computed with almost physical pion mass on very large volume shows agreement with experimental results [6]. In this paper, we present an update of our previous study [6].

For the axial-vector part of weak processes, it is known that $F_{A}\left(q^{2}\right)$ and $F_{P}\left(q^{2}\right)$ are not fully independent. It is because the axial Ward-Takahashi identity: $\partial_{\alpha} A_{\alpha}^{+}(x)=2 \hat{m} P^{+}(x)$ leads to the generalized Goldberger-Treiman (GT) relation among three form factors [7]:

$$
2 M_{N} F_{A}\left(q^{2}\right)-q^{2} F_{P}\left(q^{2}\right)=2 \hat{m} G_{P}\left(q^{2}\right),
$$

where $\hat{m}=m_{u}=m_{d}$ is a degenerate up and down quark mass and the pseudo-scalar nucleon form factor $G_{P}\left(q^{2}\right)$ is defined in the pseudo-scalar nucleon matrix element

$$
\left\langle p\left|P^{+}(x)\right| n\right\rangle=\bar{u}_{p}\left(\gamma_{5} G_{P}\left(q^{2}\right)\right) u_{n} e^{i q \cdot x}
$$

with a local pseudo-scalar density, $P^{+}(x) \equiv \bar{u}(x) \gamma_{5} d(x)$. Therefore, the $q^{2}$ dependences of three form factors, $F_{A}\left(q^{2}\right), F_{P}\left(q^{2}\right)$ and $G_{P}\left(q^{2}\right)$ are constrained by Eq. (6) as a consequence of the axial WardTakahashi identity. In this work, we also report on preliminary results of three form factors, $F_{A}\left(q^{2}\right)$, $F_{P}\left(q^{2}\right)$ and $G_{P}\left(q^{2}\right)$ in order to verify the axial Ward-Takahashi identity in terms of the nucleon matrix elements.

\section{Simulation details}

We use the 2+1 flavor QCD gauge configurations generated with the stout-smeared $O(a)$ improved Wilson-clover quark action and the Iwasaki gauge action on a $L^{3} \times T=96^{3} \times 96$ lattice at $\beta=1.82$, which corresponds to a lattice cutoff of $a^{-1} \approx 2.3 \mathrm{GeV}(a \approx 0.085 \mathrm{fm})$ [13]. The improvement coefficient, $C_{\mathrm{SW}}=1.11$, is determined nonperturbatively by the Schrödinger functional (SF) scheme. The hopping parameters for the light sea quarks $\left\{\kappa_{u d}, \kappa_{s}\right\}=\{0.126117,0.124812\}$ are chosen to be near the physical point. We calculate iso-vector nucleon form factors in the vector, axial-vector and pseudo-scalar channels with a large spatial volume of $(8.1 \mathrm{fm})^{3}$ and a simulated pion mass reaching down to $m_{\pi}=145 \mathrm{MeV}$ in $2+1$ flavor QCD. A brief summary of our simulation parameters are tabulated in Table 1. 
Table 1. Summary of simulation parameters in $2+1$ flavor QCD simulations. See Ref. [13] for further details.

\begin{tabular}{ccccccccc}
\hline$\beta$ & $L^{3} \times T$ & $C_{\mathrm{SW}}$ & $\kappa_{u d}$ & $\kappa_{s}$ & $a^{-1}[\mathrm{GeV}]$ & $(\mathrm{La})^{3}$ & $m_{\pi}[\mathrm{MeV}]$ & $N_{\text {conf }}$ \\
\hline 1.82 & $96^{3} \times 96$ & 1.11 & 0.126117 & 0.124812 & $2.333(18)$ & $\sim(8.1 \mathrm{fm})^{3}$ & 145 & 200 \\
\hline
\end{tabular}

Our preliminary results of the nucleon form factors with less number of measurements were published in Ref. [6]. The total number of gauge configurations used in the present work is 200 which corresponds to 2000 trajectories. Each measurement is separated by 10 trajectories. We use the jackknife method with a bin size of 50 trajectories for estimating the statistical errors. For technical details of how to extract the nucleon form factor from three-point functions, see Refs. [6, 7].

\section{Electric and magnetic form factors}

\subsection{Numerical results}

First of all, we show the results of iso-vector electric form factor $G_{E}$ (left panel) and magnetic form factor $G_{M}$ (right panel) in Fig. 1. For the finite three momentum $\boldsymbol{q}$, we use the nine lowest nonzero momenta: $\boldsymbol{q}=2 \pi / L \times \boldsymbol{n}$ with $|\boldsymbol{n}|^{2} \leq 9$. The lowest nonzero momentum transfer in present work reaches the value of $0.024(1)\left[(\mathrm{GeV})^{2}\right]$, which is much smaller than $4 m_{\pi}^{2}$ even at $m_{\pi}=145 \mathrm{MeV}$. In each panel, we also plot Kelly's fit [11] (red dashed curves) as their experimental data. The simulated electric form factor is fairly consistent with the experiment, especially at low $q^{2}$. The magnetic form factor agrees with the Kelly's fit albeit with rather large errors.
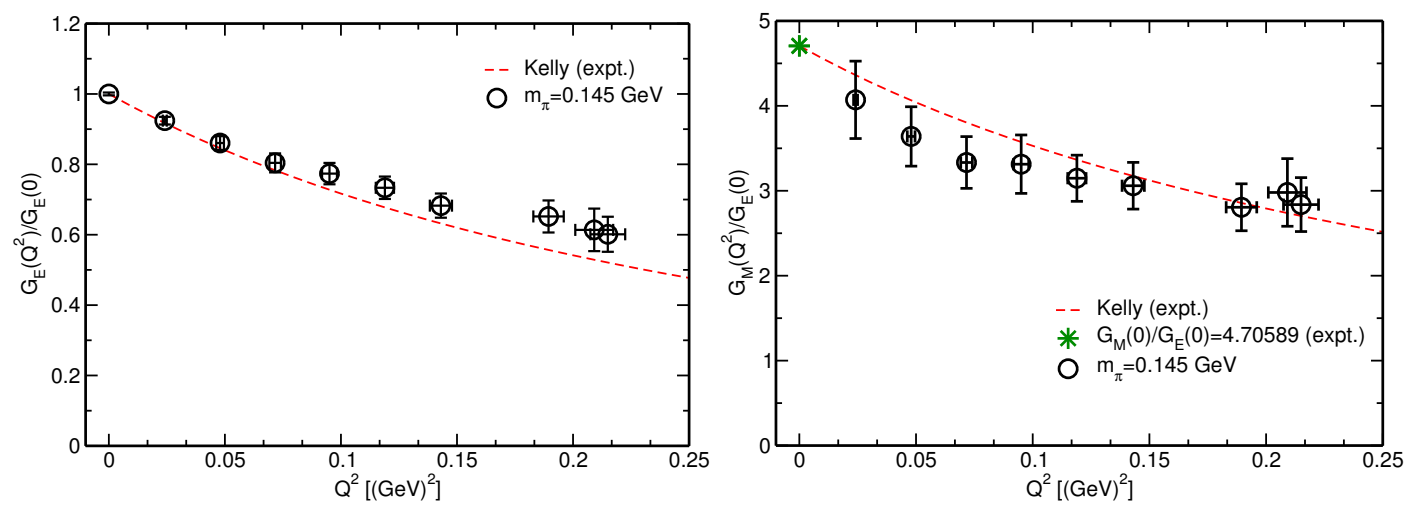

Figure 1. Momentum-squared dependence of the iso-vector electric form factor $G_{E}$ (left) and magnetic form factor $G_{M}$ (right).

The electric and magnetic RMS radii can be evaluated from the slope of the respective form factor at zero momentum transfer as described previously. Recall that $G_{M}$ at the zero momentum transfer, whose value corresponds to the iso-vector magnetic moment $\mu_{V}=G_{M}(0)$, cannot be directly measured for the kinematical reason [7]. Therefore, $q^{2}$-extrapolation is necessary to evaluate the value of $G_{M}(0)$. In principle, low $q^{2}$ data points are crucial for determination of both the RMS radii and magnetic moment. However, the accessible momentum transfer is limited on the lattice since the value of the squared three momentum transfers is discrete in units of $(2 \pi / L)^{2}$. In this sense, a large spatial size $L$ is required for precise determination of the RMS radii $\left(r_{E}\right.$ and $\left.r_{M}\right)$ and magnetic moment $\left(\mu_{V}\right)$. 


\subsection{Analysis with $z$-expansion method}

The determination of the slope (or $q^{2}$-extrapolation) is highly sensitive to how we fit the $q^{2}$-dependence of the form factors. In the previous studies [1-4], the dipole form $G\left(q^{2}\right)=a /\left(b+q^{2}\right)^{2}$ and the polynomial form $G\left(q^{2}\right)=\sum_{k=0} a_{k} q^{2 k}$ have been often adopted for $G_{E}$ and $G_{M}$. However, the fitting form ansatz may tend to constrain the interpolation (or extrapolation) and introduce a model dependence into the final result of the RMS radius (or the value of $G(0)$ ). In order to reduce systematic errors associated with an interpolation of the form factor in momentum transfer, we use the model-independent $z$-expansion method [9, 10].

Let us recall the analytic structure of the form factors in the complex $q^{2}$-plane. There is a nonanalytic region for $G\left(q^{2}\right)$ due to threshold of two or more particles, e.g. $2 \pi$ continuum. Hence the $q^{2}$-expansion, $G\left(q^{2}\right)=\sum_{k=0} a_{k} q^{2 k}$, does not converge if $\left|q^{2}\right|>4 m_{\pi}^{2}$ where $q^{2}=-4 m_{\pi}^{2}$ is a branch point associated with the pion pair creation for $G=G_{E}$ or $G_{M}$ [10]. The $z$-expansion (denoted as z-Exp) makes use of a conformal mapping from $q^{2}$ to a new variable $z$. Since this transformation makes the analytic domain mapped inside a unit-circle $|z|<1$ in the $z$-plane, the form factors can be described by a convergent Taylor series in terms of $z$ :

$$
G(z)=\sum_{k=0}^{k_{\max }} c_{k} z\left(q^{2}\right)^{k} \quad \text { with } \quad z\left(q^{2}\right)=\frac{\sqrt{4 m_{\pi}^{2}+q^{2}}-2 m_{\pi}}{\sqrt{4 m_{\pi}^{2}+q^{2}}+2 m_{\pi}} .
$$

where $k_{\max }$ truncates an infinite series expansion in $z$ and $m_{\pi}$ corresponds to the simulated pion mass. Once the ratios of $\left|c_{k} / c_{k-1}\right|$ reach a convergence value less than unity at $k<k_{\max }$, the z-Exp method makes a model independent fit and reduces systematic uncertainties to determine the RMS radius and the value of $G(0)$.
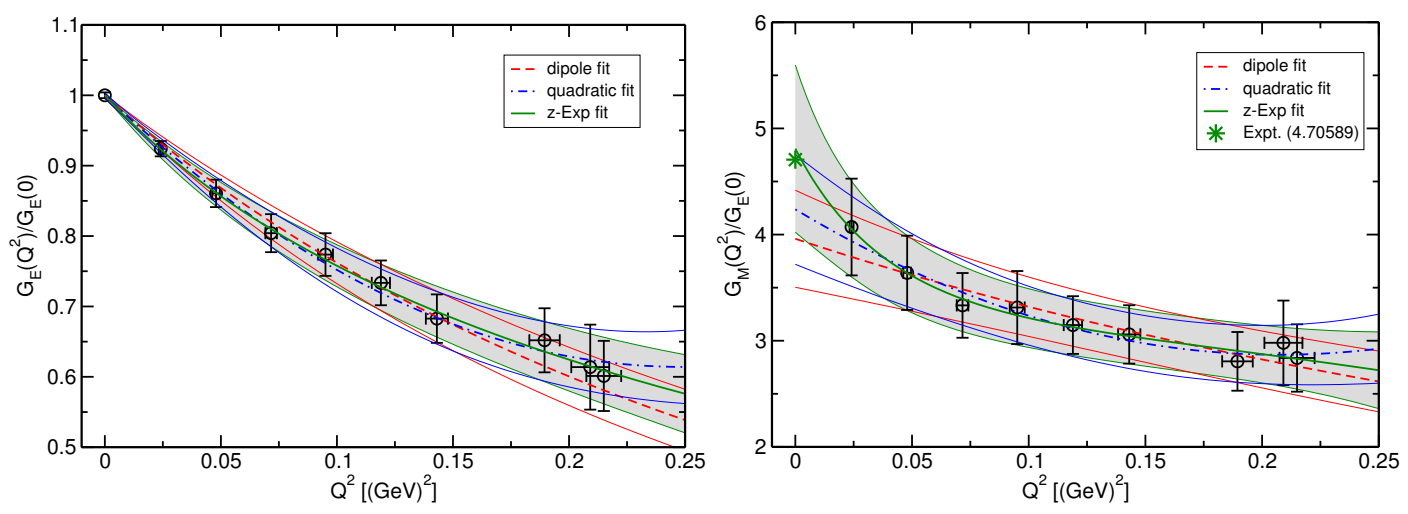

Figure 2. Results of $G_{E}$ (left panel) and $G_{M}$ (right panel) with three types of the fitting form ansatz: dipole (red), quadratic (blue) and z-Exp (green) fits. All fits are performed with all 10 (9) data points for $G_{E}\left(G_{M}\right)$.

In Fig. 2, we show the fit results of $G_{E}\left(q^{2}\right)$ (left panel) and $G_{M}\left(q^{2}\right)$ (right panel) with three types of fits: dipole (red), quadratic (blue) and z-Exp (green) fits. All the fits reasonably describe all 10 (9) data points for $G_{E}\left(G_{M}\right)$ with $\chi^{2} /$ dof $<1$. However, if one takes a closer look at low $q^{2}$, the fit curve given by the $z$-expansion fit goes nicely through the data points in low $q^{2}$ region. This implies that the z-Exp fit is relatively insensitive on the higher $q^{2}$ data points as was expected. Indeed, the z-Exp gives a rapid convergence series, which guarantees that the fit result is observed to be insensitive on 
the choice of $k_{\max }$ if $k_{\max } \geq 3$. We then obtain $r_{E}=0.917 \pm 0.099$ fm from $G_{E}$ and $\mu_{V}=4.81 \pm 0.79$ from $G_{M}$.

In Fig. 3, we compare the results from the z-Exp fit with those of dipole and quadratic fits. Although the z-Exp fit result has relatively larger error than the other results, its error may include both statistical and systematic uncertainties without any model dependence. Moreover each result of $r_{E}$ and $\mu_{V}$ from the z-Exp fit is most consistent with respective experiment. We finally compare our results with recent lattice results from PNDME [1], LHPC [2], the Mainz group [3], and ETMC [4, 5] as shown in Fig.4.
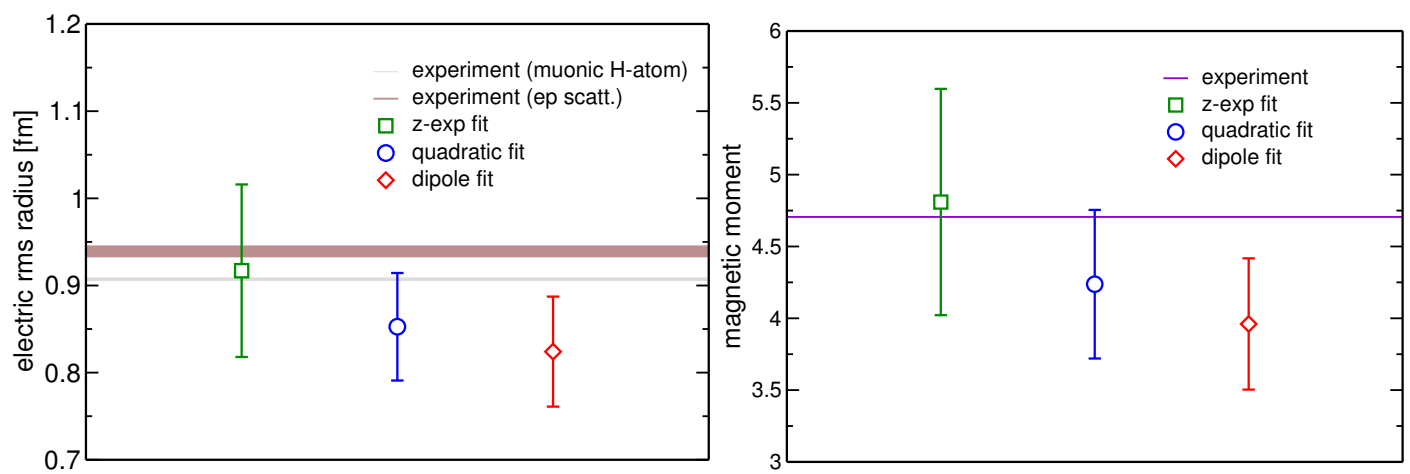

Figure 3. Comparison among results obtained with three types of the fitting form ansatz for the electric RMS radius $r_{E}$ (right panel) and magnetic moment $\mu_{V}$ (left panel). In the left panel, two horizontal bands represent experimental results from muonic $H$ atom (gray) and $e p$ scattering (brown).
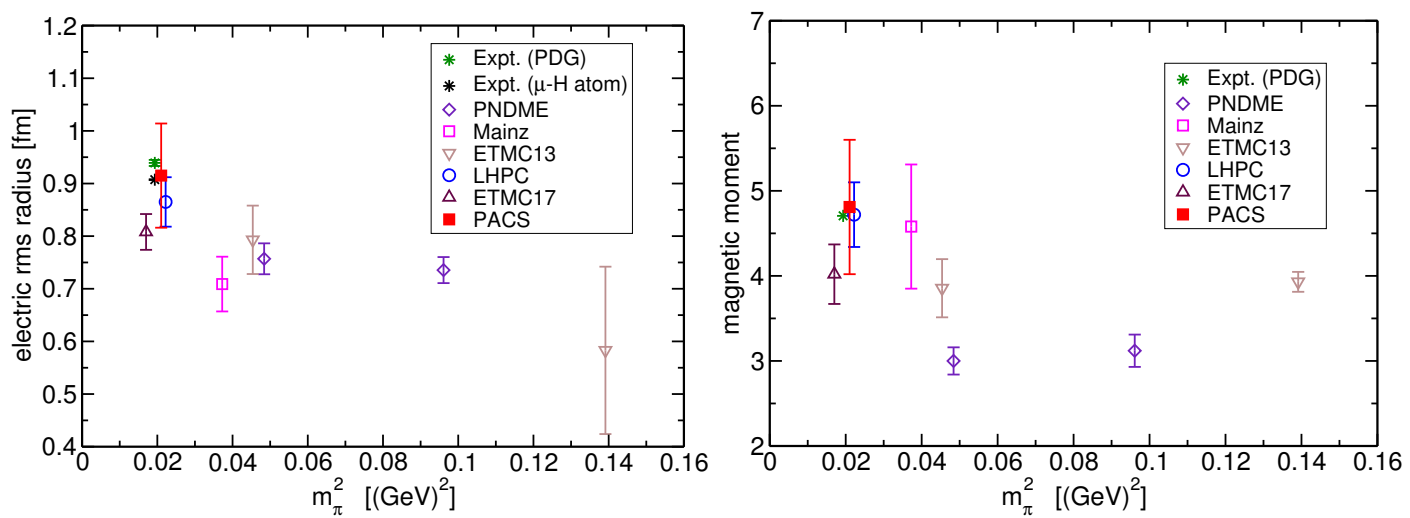

Figure 4. Our results for $r_{E}$ (left) and $\mu_{V}$ (right) at $m_{\pi}=145 \mathrm{MeV}$ (filled square) compared to recent lattice results [1-5]. The asterisk symbols represent the experimental results [8]. 


\section{Axial vector form factors}

\subsection{Numerical results}

We next show preliminary results for the nucleon form factors, $F_{A}$ and $F_{P}$, in the axial-vector channel in Fig. 5. Although $F_{A}$ is barely consistent with the experimental curve, $F_{P}$ is underestimated in comparison with both experiments and the pion-pole dominance (PPD) model. Although two experiments of muon capture and pion-photo production follow a curve given by the PPD model, where the induced pseudo-scalar form factor is given as $F_{P}^{\mathrm{PPD}}\left(q^{2}\right)=2 M_{N} F_{A}\left(q^{2}\right) /\left(q^{2}+m_{\pi}^{2}\right)$, such functional form becomes justified by the generalized GT relation (6) only in the chiral limit $(\hat{m}=0)$. However, Fig. 5 may suggest that the measured $F_{P}\left(q^{2}\right)$ does not reproduce the expected pion-pole behavior.
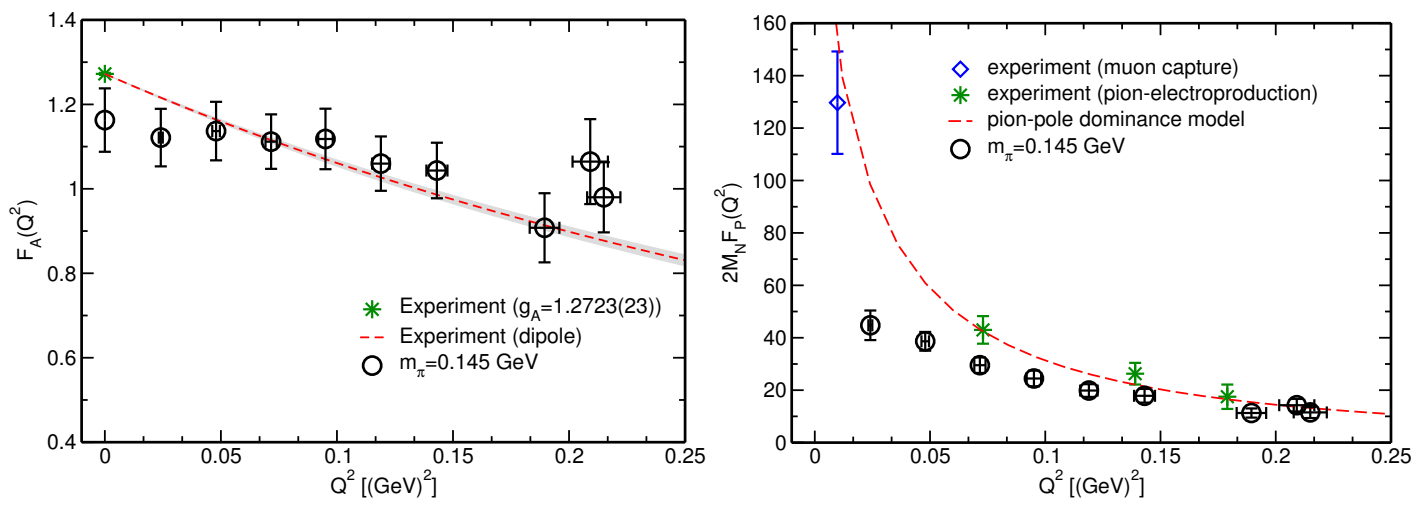

Figure 5. Momentum-squared dependence of the axial-vector from factor $F_{A}$ (left) and induced-pseudoscalar form factor $F_{P}$ (right). Both form factors are renormalized with $Z_{A}=0.9650(68)$, which is given in the SF scheme [12]. In the left panel, the experimental curve is given by a dipole form with the RMS radius of $0.66(14)$ $\mathrm{fm}$, while the red dashed curve in the right panel is given by the pion-pole dominance model.

\subsection{Test for the axial Ward-Takahashi identity}

To test whether the correct chiral behavior is well satisfied in our simulations, we also calculate the pseduo-scalar matrix element, which is described by the single form factor called as the pseduo-scalar form factor $G_{P}\left(q^{2}\right)$ defined in Eq. (7). In Fig. 6, we plot the bare pseudo-scalar form factor $G_{P}\left(q^{2}\right)$, which is not renormalized. The measured $q^{2}$ dependence of $G_{P}\left(q^{2}\right)$ resembles that of $F_{P}\left(q^{2}\right)$, where the relatively strong $q^{2}$ dependence appears in the lower $q^{2}$ region as was expected from the pionpole contribution. In order to verify the axial Ward-Takahashi identity in terms of the nucleon matrix elements, we define the following ratio inspired by the generalized GT relation (6):

$$
m_{\mathrm{AWTI}}=\frac{2 M_{N} F_{A}\left(q^{2}\right)-q^{2} F_{P}\left(q^{2}\right)}{2 G_{P}\left(q^{2}\right)}
$$

with the simulated nucleon mass $M_{N}$. If the ratio reveals a $q^{2}$ independent plateau in the entire $q^{2}$ region, $m_{\mathrm{AWTI}}$ should be regarded as a bare quark mass. As shown in Fig. 7 , the ratio $m_{\mathrm{AWTI}}$ has no appreciable $q^{2}$ dependence. It indicates that three form factors well satisfy the generalized GT relation. We can read off $a m_{\text {AWTI }} \sim 0.005$, which is more than 3 times larger than an alternative (bare) quark mass $\left(a_{\mathrm{PCAC}}=0.001577(10)\right)$ obtained from the pion two-point correlation functions with the 
partially conserved axial-vector current (PCAC) relation $[12,13]$. Since the PCAC relation is also a consequence of the axial Ward-Takahashi identity, a relation $m_{\mathrm{PCAC}} \approx m_{\mathrm{AWTI}}$ was expected. However, it is not the case. This issue may be connected with modification of the pion-pole structure in both $F_{A}\left(q^{2}\right)$ and $G_{P}\left(q^{2}\right)$. Such studies are now under way.
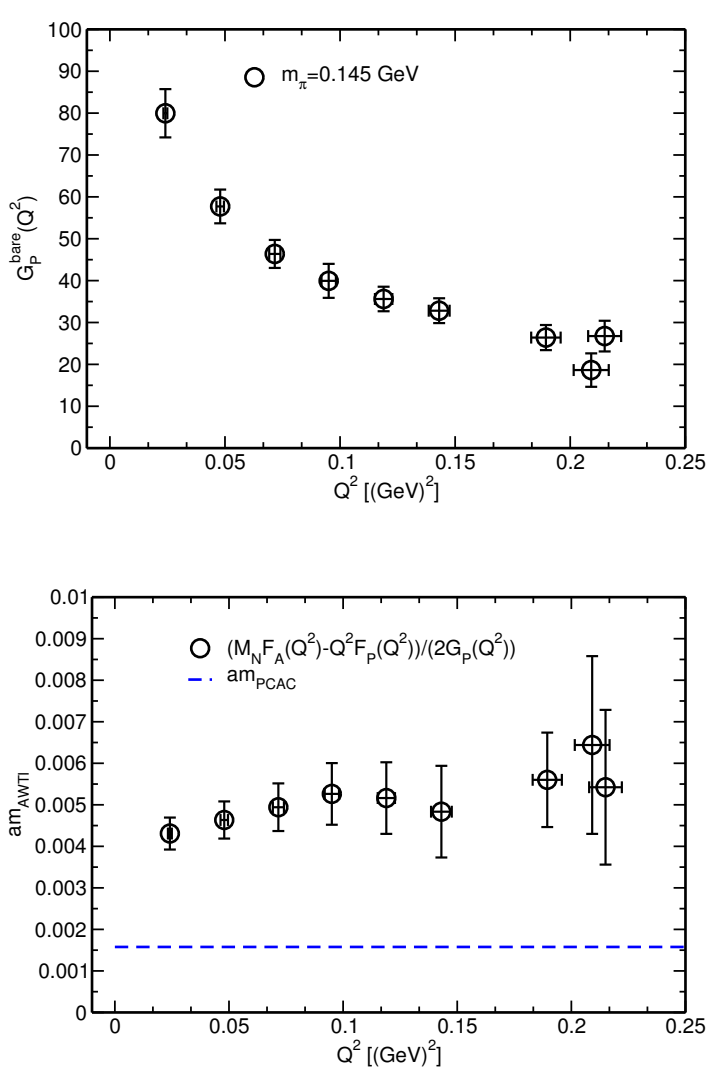

Figure 6. Momentum-squared dependence of the pseudo-scalar form factor, which is the bare value, i.e. not renormalized.
Figure 7. The ratio $m_{\mathrm{AWTI}}$ defined in Eq. (9) as a function of momentum squared $q^{2}$. A blue dashed line denotes the PCAC quark mass $[12,13]$.

\section{Summary}

We have studied the nucleon form factors calculated in $2+1$ flavor QCD near the physical point $\left(m_{\pi}\right.$ $=145 \mathrm{MeV}$ ) on a large spatial volume $(8.1 \mathrm{fm})^{3}$. We carefully examine both electric and magnetic form factor shapes with a model-independent analysis based on the $z$-expansion method. As a result, we obtain the iso-vector electric RMS radius $r_{E}$ and magnetic moment $\mu_{V}$, which are consistent with experimental values. We also analyzed axial and pseudoscalar nucleon matrix elements, which are described by $F_{A}, F_{P}$ and $G_{P}$ form factors. We have found that the axial-vector form factor $F_{A}$ is barely consistent with experiments. In addition, $q^{2}$ dependences of three form factors, $F_{A}, F_{P}$ and $G_{P}$ are well constrained by the generalized GT relation as a consequence of the axial Ward-Takahashi identity, while the induced pseudo-scalar form factor $F_{P}$ is itself underestimated in comparison with experiments in low $q^{2}$ region. 
Acknowledgments: Numerical calculations for the present work have been carried out on the FX10 supercomputer system at Information Technology Center of the University of Tokyo, on the HAPACS and COMA cluster systems under the "Interdisciplinary Computational Science Program" of Center for Computational Science at University of Tsukuba, on the Oakforest-PACS system of Joint Center for Advanced High Performance Computing, on HOKUSAI GreatWave at Advanced Center for Computing and Communication of RIKEN, and on the computer facilities of the Research Institute for Information Technology of Kyushu University. This research used computational resources of the HPCI system provided by Information Technology Center of the University of Tokyo, Institute for Information Management and Communication of Kyoto University, the Information Technology Center of Nagoya University, and RIKEN Advanced Institute for Computational Science through the HPCI System Research Project (Project ID: hp120281, hp130023, hp140209, hp140155, hp150135, hp160125, hp170022). We thank the colleagues in the PACS Collaboration for helpful discussions and providing us the code used in this work. This work is supported in part by MEXT SPIRE Field 5, and also by Grants-in-Aid for Scientific Research from the Ministry of Education, Culture, Sports, Science and Technology (No. 16H06002).

\section{References}

[1] T. Bhattacharya, S. D. Cohen, R. Gupta, A. Joseph, H. W. Lin and B. Yoon, Phys. Rev. D 89, no. 9, 094502 (2014).

[2] J. R. Green, J. W. Negele, A. V. Pochinsky, S. N. Syritsyn, M. Engelhardt and S. Krieg, Phys. Rev. D 90, 074507 (2014).

[3] S. Capitani et al., Phys. Rev. D 92, no. 5, 054511 (2015).

[4] C. Alexandrou, M. Constantinou, S. Dinter, V. Drach, K. Jansen, C. Kallidonis and G. Koutsou, Phys. Rev. D 88, no. 1, 014509 (2013).

[5] C. Alexandrou, M. Constantinou, K. Hadjiyiannakou, K. Jansen, C. Kallidonis, G. Koutsou and A. Vaquero Aviles-Casco, Phys. Rev. D 96, no. 3, 034503 (2017).

[6] Y. Kuramashi et al. [PACS Collaboration], PoS LATTICE 2016, 158 (2016).

[7] S. Sasaki and T. Yamazaki, Phys. Rev. D 78, 014510 (2008).

[8] C. Patrignani et al. [Particle Data Group], Chin. Phys. C 40, no. 10, 100001 (2016).

[9] C. G. Boyd, B. Grinstein and R. F. Lebed, Phys. Lett. B 353, 306 (1995).

[10] R. J. Hill and G. Paz, Phys. Rev. D 82, 113005 (2010).

[11] J. J. Kelly, Phys. Rev. C 70, 068202 (2004).

[12] K.-I. Ishikawa et al. [PACS Collaboration], PoS LATTICE 2015, 271 (2016).

[13] K.-I. Ishikawa et al. [PACS Collaboration], PoS LATTICE 2015, 075 (2016). 